\title{
Positional variation in grain mineral nutrients within a rice panicle and its relation to phytic acid concentration"
}

\author{
Da SU, Faisal SULTAN, Ning-chun ZHAO, Bing-ting LEI, Fu-biao WANG, Gang PAN, Fang-min CHENG ${ }^{\dagger *}$ \\ (College of Agriculture and Biotechnology, Zhejiang University, Hangzhou 310058, China) \\ †E-mail: chengfm@zju.edu.cn
}

Received June 5, 2014; Revision accepted Sept. 17, 2014; Crosschecked Oct. 11, 2014

\begin{abstract}
Six japonica rice genotypes, differing in panicle type, grain density, and phytic acid (PA) content, were applied to investigate the effect of grain position on the concentrations of major mineral nutrients and its relation to PA content and grain weight within a panicle. Grain position significantly affected the concentrations of the studied minerals in both the vertical and horizontal axes of a rice panicle. Heavy-weight grains, located on primary rachis and top rachis, generally had higher mineral concentrations, but were lower in PA concentration and molar ratios of PA/Zn, compared with the small-weight grains located on secondary rachis and bottom rachis, regardless of rice genotypes. However, on the basis of six rice genotypes, no significant correlations were found among mineral elements, PA, and grain weight. These results suggested that some desired minerals, like $\mathrm{Zn}$ and Fe, and their bioavailability, can be enhanced simultaneously by the modification of panicle patterns, and it will be helpful in the selection of rice genotypes with low PA and high mineral nutrients for further breeding strategy without sacrificing their high yields.
\end{abstract}

Key words: Grain position, Minerals, Phytic acid, Rice, Oryza sativa L.

doi:10.1631/jzus.B1400157 Document code: A CLC number: S311

\section{Introduction}

Micronutrient malnutrition, often called 'Hidden Hunger', now affects more than two billion people worldwide (McLaughlin et al., 1999). Populations in developing countries are particularly at risk as they live largely on plant-based diets without sufficient micronutrients gained from meat, fruits, or vegetables (Frank et al., 2009). The case is particularly severe for women, infants, and children (Umeta et al., 2005). It is therefore imperative to improve the nutrient composition of plant food so as to alleviate the widespread micronutrient malnutrition (Ferguson et al., 1988).

Rice is one of the major cereal crops, consumed by nearly two billion people worldwide. As a primary dietary source of energy, especially for the lowincome and rural population, rice plays an important

\footnotetext{
Corresponding author

* Project supported by the National Natural Science Foundation of China (Nos. 31071366 and 31271655)

(C) Zhejiang University and Springer-Verlag Berlin Heidelberg 2014
}

role in providing plant proteins, carbohydrates, and other nutrients (Ferguson et al., 1988). However, rice grain is relatively low in some micronutrients such as iron $(\mathrm{Fe})$, zinc $(\mathrm{Zn})$, and calcium $(\mathrm{Ca})$, compared with other staple crops such as wheat, maize, and legumes (Adeyeye et al., 2000).

In the past few decades, great efforts have been made to elucidate the effects of genotype and environment on mineral uptake and accumulation in rice plants (Schachtman and Barker, 1999). It has been demonstrated that mineral concentrations in rice grain vary greatly according to genotype, geographical location, and other environmental factors (Anandan et al., 2011). Although the extent of difference is cultivardependent, the former studies showed that grain position within a panicle had a considerable impact on grain chemical composition, like protein or microelements (Calderini and Ortiz-Monasterio, 2003; Liu et al., 2005a), suggesting that the improvement of spikelet architecture and morphological pattern is possible for obtaining the cultivars with high yield 
and balanced nutrition (Calderini and Ortiz-Monasterio, 2003).

Large variation among rice grains in a panicle is unfavorable for commercial value and quality (Jeng et al., 2006). Some research has been performed on the effect of grain position within a panicle on rice quality, so as to reduce the variation through improved agronomic practices, including application of plant growth regulators, and better water and fertilizer management (Jongkaewwattana and Geng, 1991). It was reported that there were large variations in grain quality (chalky occurrence, palatability, amylose, protein, and amylographic characteristics) between primary and secondary rachides (Matsue et al., 1995) and among the different positions within a rice panicle (Kobayasi et al., 2002). However, to date, little research has been done on the positional variation of micronutrient concentration in relation to rice panicle morphology.

Phytic acid (PA) in crops, including cereals and legumes, is an anti-nutritional factor for animals and human, as it can strongly chelate mineral nutrients such as $\mathrm{Zn}, \mathrm{Fe}, \mathrm{Ca}$, and magnesium $(\mathrm{Mg}$ ) (Raboy, 2001), leading to a marked reduction in their bioavailability (Raboy et al., 1984). Moreover, animals possibly excrete more phytates if they are given high PA feed, which poses a threat of contaminating water. Therefore, it was important to reduce PA concentration in rice grain for the improvement of crops' nutritional quality (Raboy, 2001).

In this experiment, six japonica rice genotypes, differing in panicle type, grain density, and PA content, were used with the following objectives: (1) to investigate the effect of grain position on mineral elements (potassium $(\mathrm{K}), \mathrm{Mg}$, sodium $(\mathrm{Na}), \mathrm{Ca}, \mathrm{Zn}$, $\mathrm{Fe}$, manganese $(\mathrm{Mn})$, and copper $(\mathrm{Cu})$ ), as well as PA concentration; (2) to identify the positional distribution of mineral elements in grains within a panicle as affected by the panicle morphology of rice genotypes; and, (3) to illustrate the relationships among mineral elements, PA content, and grain weight at different positions within a panicle.

\section{Materials and methods}

\subsection{Plant materials and field experiments}

According to previous surveys on grain PA and panicle type of 56 rice cultivars and 32 breeding lines, collected from different locations of China (Liu et al., 2005a; Cheng et al., 2007), six japonica rice genotypes (98110, HIPj, Xiushui63, Xiushui994, Chunjiang15, and Xiushui11) were selected for the current study. All genotypes were seeded in late May and transplanted in late June at the experimental farm of the Huajiachi Campus of Zhejiang University, China. Each genotype was grown in two replicates of an eight-row plot, $2 \mathrm{~m}$ long and $20 \mathrm{~cm}$ wide between rows with nine hills in each row and three seedlings per hill. The trial was managed according to locally recommended agronomic practices.

\subsection{Grain sampling and analysis}

Grains from six positions within a panicle, i.e., top primary, top secondary, middle primary, middle secondary, bottom primary, and bottom secondary rachides, were sampled for analysis. The classification of these positions for each genotype is shown in Table 1. Panicle traits, including panicle length and bending degree of panicle (an angle from the tip of panicle to the extending top of stem), were determined at maturity with 20 individual measurements of main stems in each plot (Table 1).

At maturity, 30 panicles were harvested from each replication. After being dried naturally, rice grains were collected separately from six positions, then husked, milled, and passed through a $0.5-\mathrm{mm}$ sieve. The flour was then stored in a desiccator until analysis.

The concentrations of $\mathrm{K}, \mathrm{Na}, \mathrm{Mg}, \mathrm{Ca}, \mathrm{Zn}, \mathrm{Fe}$, $\mathrm{Mn}$, and $\mathrm{Cu}$ were determined by an atomic absorption spectrophotometer (Perkin-Elmer Model AA6300) after wet digestion with nitric acid. PA was determined as described by Miller et al. (1980) with modification (Liu et al., 2005a). Triplicate measurements were performed for each sample.

\subsection{Data analysis}

Statistical analysis was performed using statistics software version SPSS 11.0. The data were submitted for variance analysis and the means were tested by least significant difference. Correlation analysis was conducted to characterize the relationships among eight mineral elements, PA content, and grain weight for different grain positions and six genotypes, respectively. 
Table 1 Classification of grain position within a panicle and panicle morphological traits for six rice cultivars

\begin{tabular}{|c|c|c|c|c|c|c|c|c|c|}
\hline \multirow[b]{2}{*}{ Cultivar } & \multirow{2}{*}{$\begin{array}{c}\text { Total number } \\
\text { of primary } \\
\text { branches }\end{array}$} & \multicolumn{3}{|c|}{ Classification of rachis within a panicle } & \multicolumn{5}{|c|}{ Panicle morphological character } \\
\hline & & $\begin{array}{l}\text { Upper } \\
\text { rachis }\end{array}$ & $\begin{array}{c}\text { Middle } \\
\text { rachis }\end{array}$ & $\begin{array}{l}\text { Lower } \\
\text { rachis }\end{array}$ & $\begin{array}{l}\mathrm{PL} \\
(\mathrm{cm})\end{array}$ & GPP & $\begin{array}{c}\text { GD } \\
\text { (grain/cm) }\end{array}$ & $\begin{array}{l}\mathrm{BD} \\
\left({ }^{\circ}\right)\end{array}$ & $\begin{array}{c}\text { Yield } \\
\left(\mathrm{kg} / \mathrm{hm}^{2}\right)\end{array}$ \\
\hline 98110 & 14 & 4 & 5 & 5 & 16.2 & 151.2 & 9.3 & 9.0 & 8427.5 \\
\hline HIPj & 14 & 4 & 5 & 5 & 16.0 & 148.7 & 9.3 & 9.2 & 8132.7 \\
\hline Xiushui63 & 12 & 3 & 5 & 4 & 15.8 & 126.5 & 8.0 & 9.7 & 7737.1 \\
\hline Xiushui994 & 14 & 4 & 5 & 5 & 15.4 & 132. 6 & 8.6 & 7.8 & 7945.3 \\
\hline Chunjiang15 & 12 & 4 & 4 & 4 & 21.5 & 103.7 & 4.8 & 76.2 & 6863.8 \\
\hline Xiushui11 & 12 & 4 & 4 & 4 & 19.2 & 110.0 & 5.7 & 86.1 & 7142.0 \\
\hline
\end{tabular}

PL: panicle length; GPP: grains per panicle; GD: grain density; BD: bending degree of panicle

\section{Results}

3.1 Positional variation in $\mathrm{K}, \mathrm{Mg}, \mathrm{Na}, \mathrm{Ca}, \mathrm{Zn}, \mathrm{Fe}$, $\mathrm{Mn}$, and $\mathrm{Cu}$ concentrations within a panicle

As shown in Table 2, grain position was an important source of variation for grain mineral concentrations. Of all eight minerals, there were significant differences among grains located on the different positions within a panicle. The grains on the top primary and middle primary rachides had substantially higher minerals concentrations than those on the bottom secondary and middle secondary rachides. The order of positional differences in mineral concentrations was: top primary rachides $>$ middle primary rachides $>$ bottom primary rachides/top secondary rachides $>$ middle secondary rachides $>$ bottom secondary rachides, although the extent of difference was genotype-dependent and varied largely with various mineral elements. This result clearly indicated that grain position affected the concentrations of all eight minerals in both vertical and horizontal axes of the rice panicle. In comparative terms, the effects of grain positions on $\mathrm{Na}, \mathrm{Fe}$, and $\mathrm{Cu}$ concentrations were more substantial than those on $\mathrm{Ca}$ and $\mathrm{Zn}$ concentrations, as reflected by their differences in coefficients of variation (CVs) and the variable range of different positions (Table 2).

The ratio of the eight mineral concentrations was relatively stable or showed only slight variations among different grain positions in a rice cultivar (Table 2). However, relatively great variability in the ratio of various minerals was found among cultivars, although the concentrations of $\mathrm{Zn}, \mathrm{Fe}, \mathrm{Mn}$, and $\mathrm{Cu}$ were found to be remarkably lower than those of $\mathrm{K}$, $\mathrm{Mg}, \mathrm{Na}$, and $\mathrm{Ca}$ in rice grains, with the lowest values for $\mathrm{Cu}$ and $\mathrm{Fe}$, and the highest for $\mathrm{K}$ and $\mathrm{Mg}$.
For instance, 98110 had much higher $\mathrm{Ca}$ and $\mathrm{Mg}$ concentrations than Xiushui11, whereas the concentrations of $\mathrm{Fe}, \mathrm{Mn}$, and $\mathrm{Cu}$ for 98110 were lower than those for Xiushui11, in terms of an average of six different positions. This result suggested that grain positions had a relatively small impact on the ratio of mineral concentrations compared with the rice genotypes, despite the different sensitivities to the effect of grain positions among different mineral elements.

Considering the yield potential of rice genotypes (Table 1) and grain mineral concentrations (Table 2), little relationship was found between the eight mineral concentrations and rice genotypes, differing in grain density in a panicle and grain yield, in terms of average different positions. In contrast, the extent of positional variation in some mineral elements $(\mathrm{K}, \mathrm{Na}$, $\mathrm{Fe}$, and $\mathrm{Cu}$ ), particularly the variable range between top primary rachides and bottom secondary rachides, even tended to be more pronounced than that of the six rice genotype variations (Table 2). This implied that the concentrations of mineral elements in rice grains were not inherently related to the level of grain yield for different rice genotypes.

\subsection{Positional variation in PA content, grain weight, molar ratios of $\mathrm{PA} / \mathrm{Zn}$ and $\mathrm{PA} / \mathrm{Fe}$ within a panicle}

From Table 3, the marked differences among different positions were found in PA concentration, grain weight, and molar ratios of $\mathrm{PA} / \mathrm{Zn}$ and $\mathrm{PA} / \mathrm{Fe}$. For grain weight, the grains located on the primary rachis (PR) and top rachis (TR) were significantly heavier than those on the secondary rachis (SR) and bottom rachis (BR), irrespective of rice genotype. On the other hand, the grains on PR and TR had 
Table 2 Positional variations in the concentrations of $\mathrm{K}, \mathrm{Mg}, \mathrm{Na}, \mathrm{Ca}, \mathrm{Zn}, \mathrm{Fe}, \mathrm{Mn}$, and $\mathrm{Cu}$ in six different rice cultivars

\begin{tabular}{|c|c|c|c|c|c|c|c|c|c|}
\hline \multirow{2}{*}{ Cultivar } & \multirow{2}{*}{$\begin{array}{c}\text { Grain } \\
\text { position }\end{array}$} & \multicolumn{8}{|c|}{ Concentration $(\mu \mathrm{g} / \mathrm{g})$} \\
\hline & & $\mathrm{K}$ & $\mathrm{Mg}$ & $\mathrm{Na}$ & $\mathrm{Ca}$ & $\mathrm{Zn}$ & $\mathrm{Fe}$ & $\mathrm{Mn}$ & $\mathrm{Cu}$ \\
\hline \multirow[t]{8}{*}{98110} & TPR & $1627 \mathrm{a}$ & $1518 \mathrm{a}$ & $260.6 \mathrm{a}$ & $263.7 \mathrm{a}$ & $25.28 \mathrm{a}$ & $10.27 \mathrm{a}$ & $24.82 \mathrm{bc}$ & $2.780 \mathrm{a}$ \\
\hline & TSR & $1568 \mathrm{~b}$ & $1453 \mathrm{ab}$ & $219.8 \mathrm{ab}$ & $256.5 \mathrm{~b}$ & $24.58 b$ & $9.57 \mathrm{c}$ & $24.20 \mathrm{c}$ & $2.471 b$ \\
\hline & MPR & $1468 \mathrm{c}$ & $1398 b$ & $203.3 b c$ & $253.9 b c$ & $24.55 b$ & $9.89 b$ & $26.08 \mathrm{a}$ & $2.425 b$ \\
\hline & MSR & 1396d & $1315 \mathrm{c}$ & $191.7 \mathrm{bcd}$ & $250.7 \mathrm{bc}$ & $24.07 \mathrm{c}$ & $9.31 \mathrm{~d}$ & $26.03 \mathrm{a}$ & $2.273 b$ \\
\hline & BPR & $1440 \mathrm{~cd}$ & $1378 \mathrm{bc}$ & $170.4 \mathrm{~cd}$ & $247.8 \mathrm{~cd}$ & $23.48 \mathrm{~d}$ & $8.96 \mathrm{e}$ & $25.19 b$ & $1.866 \mathrm{c}$ \\
\hline & BSR & $1314 \mathrm{e}$ & $1324 \mathrm{c}$ & $156.3 d$ & $242.3 d$ & $22.78 \mathrm{e}$ & $8.59 \mathrm{f}$ & $24.63 c$ & $1.671 \mathrm{c}$ \\
\hline & Mean & 1469 & 1398 & 200.4 & 252.5 & 24.12 & 9.43 & 25.16 & 2.248 \\
\hline & $\mathrm{CV}$ & $7.76 \%$ & $5.56 \%$ & $18.57 \%$ & $2.93 \%$ & $3.69 \%$ & $6.49 \%$ & $3.04 \%$ & $18.28 \%$ \\
\hline \multirow[t]{8}{*}{$\mathrm{HIPj}$} & TPR & $1862 \mathrm{a}$ & $1704 a$ & $265.6 \mathrm{a}$ & $282.1 \mathrm{a}$ & $26.76 \mathrm{a}$ & $11.93 \mathrm{a}$ & $31.62 \mathrm{a}$ & $2.815 \mathrm{a}$ \\
\hline & TSR & $1732 b$ & $1585 b$ & 200.5 cde & $269.4 \mathrm{~b}$ & $25.97 b$ & $10.90 \mathrm{c}$ & $28.78 b$ & $2.567 \mathrm{bc}$ \\
\hline & MPR & $1583 \mathrm{c}$ & $1440 \mathrm{c}$ & $241.7 \mathrm{ab}$ & $267.7 \mathrm{~b}$ & $25.83 b$ & $11.33 b$ & $27.52 \mathrm{c}$ & $2.688 \mathrm{ab}$ \\
\hline & MSR & $1285 \mathrm{e}$ & $1321 d$ & $215.1 b c$ & $263.8 \mathrm{c}$ & $25.29 \mathrm{c}$ & $10.92 \mathrm{c}$ & $25.55 \mathrm{c}$ & $2.459 \mathrm{~cd}$ \\
\hline & BPR & $1536 \mathrm{~d}$ & $1389 \mathrm{c}$ & 192.6de & $257.6 \mathrm{~d}$ & $24.75 d$ & $10.42 \mathrm{~d}$ & $26.81 \mathrm{c}$ & $2.302 \mathrm{~d}$ \\
\hline & BSR & $1328 \mathrm{e}$ & $1329 d$ & $184.1 \mathrm{e}$ & $253.1 \mathrm{e}$ & $24.38 \mathrm{~d}$ & $9.88 \mathrm{e}$ & $25.07 \mathrm{~d}$ & $2.022 \mathrm{e}$ \\
\hline & Mean & 1554 & 1461 & 216.6 & 265.6 & 25.50 & 10.90 & 27.56 & 2.476 \\
\hline & $\mathrm{CV}$ & $14.43 \%$ & $10.47 \%$ & $14.49 \%$ & $3.83 \%$ & $3.41 \%$ & $6.53 \%$ & $8.71 \%$ & $11.49 \%$ \\
\hline \multirow[t]{8}{*}{ Xiushui63 } & TPR & $1836 a$ & $1757 \mathrm{a}$ & $312.2 \mathrm{a}$ & $290.1 \mathrm{a}$ & $22.87 \mathrm{a}$ & $9.68 \mathrm{a}$ & $46.83 a$ & $2.505 \mathrm{a}$ \\
\hline & TSR & $1790 \mathrm{a}$ & $1671 \mathrm{ab}$ & $267.7 b$ & $283.2 b$ & $22.50 \mathrm{a}$ & $9.42 \mathrm{bc}$ & $43.27 \mathrm{c}$ & $2.167 \mathrm{~b}$ \\
\hline & MPR & $1802 \mathrm{a}$ & $1655 b$ & $275.8 b$ & $281.8 b$ & $21.99 b$ & $9.33 \mathrm{~cd}$ & $45.03 b$ & $2.225 b$ \\
\hline & MSR & $1570 \mathrm{~b}$ & $1415 \mathrm{c}$ & $249.9 b c$ & $276.9 \mathrm{c}$ & $21.28 \mathrm{c}$ & $9.16 \mathrm{~d}$ & $38.87 \mathrm{de}$ & $2.168 b$ \\
\hline & BPR & $1737 \mathrm{a}$ & $1715 \mathrm{ab}$ & $209.7 \mathrm{c}$ & $270.3 d$ & $20.88 \mathrm{~cd}$ & $8.70 \mathrm{e}$ & $42.87 \mathrm{c}$ & $2.010 \mathrm{~b}$ \\
\hline & BSR & $1498 b$ & $1429 c$ & $191.9 \mathrm{c}$ & $262.4 \mathrm{e}$ & $20.44 d$ & $8.40 \mathrm{f}$ & $38.73 \mathrm{e}$ & $1.643 \mathrm{c}$ \\
\hline & Mean & 1706 & 1607 & 251.2 & 277.5 & 21.66 & 9.11 & 42.60 & 2.120 \\
\hline & $\mathrm{CV}$ & $8.12 \%$ & $9.19 \%$ & $17.66 \%$ & $3.57 \%$ & $4.39 \%$ & $5.25 \%$ & $7.66 \%$ & $13.40 \%$ \\
\hline \multirow[t]{8}{*}{ Xiushui994 } & TPR & $1655 a$ & $1359 a$ & $312.2 \mathrm{a}$ & $304.2 \mathrm{a}$ & $20.84 a$ & $10.81 \mathrm{a}$ & $36.88 \mathrm{a}$ & $3.270 \mathrm{a}$ \\
\hline & TSR & $1587 \mathrm{ab}$ & $1297 \mathrm{ab}$ & $239.1 b$ & $296.1 b$ & $20.46 a b$ & $10.18 b$ & $32.36 \mathrm{c}$ & $2.908 \mathrm{~b}$ \\
\hline & MPR & $1492 b$ & $1305 \mathrm{ab}$ & $218.4 b$ & $292.3 \mathrm{c}$ & $19.89 \mathrm{ab}$ & $10.23 b$ & $33.81 \mathrm{~b}$ & $2.868 \mathrm{~b}$ \\
\hline & MSR & $1571 \mathrm{ab}$ & $1245 b$ & $202.2 \mathrm{bc}$ & $287.4 d$ & $19.75 \mathrm{ab}$ & $9.76 \mathrm{c}$ & $32.52 \mathrm{c}$ & $2.673 b c$ \\
\hline & BPR & $1514 \mathrm{~b}$ & $1291 \mathrm{ab}$ & $167.6 \mathrm{~cd}$ & $282.7 \mathrm{e}$ & $19.21 \mathrm{~b}$ & $9.17 \mathrm{~d}$ & $32.98 \mathrm{c}$ & $2.442 \mathrm{~cd}$ \\
\hline & BSR & $1465 b$ & $1225 b$ & $150.6 \mathrm{~d}$ & $276.1 \mathrm{f}$ & $18.79 b$ & $8.96 \mathrm{e}$ & $30.29 \mathrm{~d}$ & $2.198 \mathrm{~d}$ \\
\hline & Mean & 1547 & 1287 & 215.0 & 289.8 & 19.82 & 9.85 & 33.14 & 2.727 \\
\hline & $\mathrm{CV}$ & $4.54 \%$ & $3.68 \%$ & $26.80 \%$ & $3.44 \%$ & $3.84 \%$ & $7.09 \%$ & $6.55 \%$ & $13.84 \%$ \\
\hline \multirow[t]{8}{*}{ Chunjiang15 } & TPR & $1571 \mathrm{a}$ & $1456 \mathrm{a}$ & $228.9 \mathrm{a}$ & $254.0 \mathrm{a}$ & $24.81 \mathrm{a}$ & $9.72 \mathrm{a}$ & $34.02 \mathrm{a}$ & $3.208 \mathrm{a}$ \\
\hline & TSR & $1308 b c$ & $1339 b$ & $179.9 \mathrm{bc}$ & $247.8 b$ & $22.40 \mathrm{~b}$ & $9.26 \mathrm{~b}$ & $32.30 \mathrm{~b}$ & $2.975 b$ \\
\hline & MPR & $1395 b$ & $1310 \mathrm{bc}$ & $202.0 \mathrm{abc}$ & $247.0 \mathrm{~b}$ & $21.55 \mathrm{c}$ & $9.14 b$ & $31.93 b$ & $2.832 \mathrm{~b}$ \\
\hline & MSR & $1336 \mathrm{bc}$ & $1250 \mathrm{c}$ & $218.7 \mathrm{ab}$ & $240.2 \mathrm{~cd}$ & $22.40 \mathrm{~b}$ & $8.81 \mathrm{c}$ & $31.22 \mathrm{bc}$ & $2.870 \mathrm{~b}$ \\
\hline & BPR & $1278 \mathrm{c}$ & $1274 b c$ & $187.0 \mathrm{abc}$ & $236.9 d$ & $21.26 \mathrm{c}$ & $8.40 \mathrm{~d}$ & $30.84 \mathrm{c}$ & $2.603 \mathrm{c}$ \\
\hline & BSR & $1285 \mathrm{c}$ & $1375 b$ & $174.9 \mathrm{c}$ & $231.6 \mathrm{e}$ & $20.45 d$ & $8.18 \mathrm{e}$ & $30.18 \mathrm{c}$ & $2.525 \mathrm{c}$ \\
\hline & Mean & 1362 & 1334 & 198.7 & 242.9 & 22.15 & 8.92 & 31.75 & 2.836 \\
\hline & $\mathrm{CV}$ & $8.13 \%$ & $5.59 \%$ & $10.99 \%$ & $3.37 \%$ & $6.77 \%$ & $6.41 \%$ & $4.24 \%$ & $8.78 \%$ \\
\hline \multirow[t]{8}{*}{ Xiushui11 } & TPR & $1527 \mathrm{a}$ & $1458 \mathrm{a}$ & $273.8 \mathrm{a}$ & $229.6 \mathrm{a}$ & $24.48 \mathrm{a}$ & $11.71 \mathrm{a}$ & $30.58 \mathrm{a}$ & $2.868 \mathrm{a}$ \\
\hline & TSR & $1347 \mathrm{bc}$ & $1343 a$ & $228.2 b$ & $223.9 b$ & $23.74 \mathrm{a}$ & $11.42 \mathrm{~b}$ & $26.95 b$ & $2.672 b$ \\
\hline & MPR & $1421 \mathrm{ab}$ & $1333 a$ & $228.9 b$ & $218.8 \mathrm{~cd}$ & $24.04 \mathrm{a}$ & $11.20 \mathrm{c}$ & $25.15 b c$ & $2.568 \mathrm{c}$ \\
\hline & MSR & $1345 b c$ & $1365 \mathrm{a}$ & $216.1 b c$ & $214.6 \mathrm{~d}$ & $23.49 \mathrm{a}$ & $10.86 \mathrm{~d}$ & $24.30 \mathrm{c}$ & $2.458 \mathrm{~d}$ \\
\hline & BPR & $1276 \mathrm{c}$ & $1368 \mathrm{a}$ & $206.0 \mathrm{bc}$ & $209.5 \mathrm{e}$ & $22.33 b$ & $10.34 \mathrm{e}$ & $24.06 \mathrm{c}$ & $2.283 \mathrm{e}$ \\
\hline & BSR & $1098 \mathrm{~d}$ & $864 b$ & $191.5 \mathrm{c}$ & $203.2 \mathrm{f}$ & $21.92 b$ & $10.08 \mathrm{f}$ & $22.65 d$ & $2.132 \mathrm{f}$ \\
\hline & Mean & 1336 & 1289 & 224.1 & 216.6 & 23.33 & 10.94 & 25.62 & 2.497 \\
\hline & $\mathrm{CV}$ & $10.81 \%$ & $16.50 \%$ & $12.57 \%$ & $4.43 \%$ & $4.29 \%$ & $5.78 \%$ & $10.99 \%$ & $10.65 \%$ \\
\hline
\end{tabular}

TPR: top primary rachis; TSR: top secondary rachis; MPR: middle primary rachis; MSR: middle secondary rachis; BPR: bottom primary rachis; BSR: bottom secondary rachis; CV: coefficient of variation. The values followed by the same letters within a column are not significantly different in term of the same cultivar $(P>0.05)$ 
Table 3 Positional variations in phytic acid (PA) concentration, grain weight, and the molar ratios of PA/Zn and $\mathrm{PA} / \mathrm{Fe}$ in six different rice cultivars

\begin{tabular}{|c|c|c|c|c|c|c|c|c|c|}
\hline \multirow{2}{*}{ Cultivar } & \multirow{2}{*}{$\begin{array}{c}\text { Grain } \\
\text { position }\end{array}$} & \multicolumn{2}{|c|}{ PA concentration $(\mathrm{mg} / \mathrm{g})$} & \multicolumn{2}{|c|}{ Grain weight (mg) } & \multicolumn{2}{|c|}{$\mathrm{PA} / \mathrm{Zn}$ molar ratio } & \multicolumn{2}{|c|}{$\mathrm{PA} / \mathrm{Fe}$ molar ratio } \\
\hline & & PR & $\mathrm{SR}$ & PR & SR & PR & SR & PR & SR \\
\hline \multirow[t]{6}{*}{98110} & TR & $4.68 b$ & $4.55 b$ & $25.47 \mathrm{a}$ & $20.12 c$ & $18.35 \mathrm{c}$ & $18.34 \mathrm{c}$ & $45.17 \mathrm{e}$ & $47.09 \mathrm{~d}$ \\
\hline & MR & $4.89 \mathrm{a}$ & $4.49 \mathrm{~b}$ & $24.35 \mathrm{a}$ & $20.63 c$ & $19.74 b$ & $18.50 \mathrm{c}$ & $49.00 \mathrm{c}$ & $47.81 \mathrm{~cd}$ \\
\hline & $\mathrm{BR}$ & $5.07 \mathrm{a}$ & $5.25 \mathrm{a}$ & $22.16 b$ & $17.06 \mathrm{~d}$ & $21.39 \mathrm{a}$ & $22.85 \mathrm{a}$ & $56.02 b$ & $60.58 \mathrm{a}$ \\
\hline & Mean 1 & 4.88 & 4.76 & 23.99 & 19.27 & 19.83 & 19.89 & 50.06 & 51.83 \\
\hline & Mean 2 & \multicolumn{2}{|c|}{4.82} & \multicolumn{2}{|c|}{21.63} & \multicolumn{2}{|c|}{19.86} & \multicolumn{2}{|c|}{50.95} \\
\hline & $\mathrm{CV}$ & \multicolumn{2}{|c|}{$6.24 \%$} & \multicolumn{2}{|c|}{$14.10 \%$} & \multicolumn{2}{|c|}{$9.48 \%$} & \multicolumn{2}{|c|}{$11.80 \%$} \\
\hline \multirow[t]{6}{*}{$\mathrm{HIPj}$} & TR & $4.54 \mathrm{a}$ & $4.43 \mathrm{ab}$ & $24.12 \mathrm{a}$ & $19.05 \mathrm{~d}$ & $16.82 \mathrm{a}$ & $16.90 \mathrm{a}$ & $37.73 \mathrm{c}$ & $40.26 b$ \\
\hline & MR & $4.18 \mathrm{c}$ & $4.32 \mathrm{ab}$ & $23.16 b$ & $19.01 \mathrm{~d}$ & $16.03 b$ & $16.91 \mathrm{a}$ & $36.54 \mathrm{~cd}$ & $39.17 b$ \\
\hline & $\mathrm{BR}$ & $3.68 \mathrm{~d}$ & $4.23 \mathrm{c}$ & $20.50 \mathrm{c}$ & $18.09 \mathrm{e}$ & $14.73 \mathrm{c}$ & $17.17 \mathrm{a}$ & $35.00 \mathrm{~d}$ & $42.39 \mathrm{a}$ \\
\hline & Mean 1 & 4.13 & 4.32 & 22.59 & 18.72 & 15.86 & 16.99 & 36.42 & 40.61 \\
\hline & Mean 2 & \multicolumn{2}{|c|}{4.23} & \multicolumn{2}{|c|}{20.65} & \multicolumn{2}{|c|}{16.43} & \multicolumn{2}{|c|}{38.52} \\
\hline & $\mathrm{CV}$ & \multicolumn{2}{|c|}{$7.10 \%$} & \multicolumn{2}{|c|}{$11.89 \%$} & \multicolumn{2}{|c|}{$5.57 \%$} & \multicolumn{2}{|c|}{$6.90 \%$} \\
\hline \multirow[t]{6}{*}{ Xiushui63 } & TR & $5.64 b c$ & $5.60 \mathrm{c}$ & $26.54 \mathrm{a}$ & $23.04 \mathrm{c}$ & $24.66 \mathrm{~d}$ & $25.24 d$ & $58.26 \mathrm{c}$ & $60.30 \mathrm{c}$ \\
\hline & MR & $6.13 \mathrm{a}$ & $6.06 \mathrm{a}$ & $25.68 \mathrm{ab}$ & $22.40 \mathrm{c}$ & $27.88 \mathrm{c}$ & $28.48 b$ & $65.70 \mathrm{~b}$ & $66.16 \mathrm{~b}$ \\
\hline & $\mathrm{BR}$ & $5.97 \mathrm{ab}$ & $5.98 \mathrm{ab}$ & $25.26 b$ & $22.45 \mathrm{c}$ & $28.59 b$ & $29.26 \mathrm{a}$ & $68.62 b$ & $71.19 \mathrm{a}$ \\
\hline & Mean 1 & 5.91 & 5.88 & 25.83 & 22.63 & 27.04 & 27.66 & 64.19 & 65.88 \\
\hline & Mean 2 & & & & & & & & \\
\hline & $\mathrm{CV}$ & & & & $8 \%$ & & $1 \%$ & & $6 \%$ \\
\hline Xiushui994 & TR & $4.58 \mathrm{ab}$ & $4.46 b$ & $25.71 \mathrm{a}$ & $23.18 \mathrm{ab}$ & $21.98 \mathrm{c}$ & $21.80 \mathrm{c}$ & $42.37 \mathrm{~d}$ & $43.81 \mathrm{c}$ \\
\hline & MR & $4.28 \mathrm{c}$ & $4.36 \mathrm{c}$ & $24.14 \mathrm{a}$ & $18.62 \mathrm{c}$ & $21.52 \mathrm{c}$ & $22.07 \mathrm{c}$ & $41.84 \mathrm{~d}$ & $44.67 \mathrm{c}$ \\
\hline & $\mathrm{BR}$ & $4.43 \mathrm{bc}$ & $4.64 \mathrm{a}$ & $20.29 b c$ & $17.57 \mathrm{c}$ & $23.06 \mathrm{~b}$ & $24.69 \mathrm{a}$ & $48.31 \mathrm{~b}$ & $51.78 \mathrm{a}$ \\
\hline & Mean 1 & 4.43 & 4.49 & 23.28 & 19.79 & 22.19 & 22.85 & 44.17 & 46.75 \\
\hline & Mean 2 & & & & & & & & \\
\hline & $\mathrm{CV}$ & & & & $4 \%$ & & $7 \%$ & & $7 \%$ \\
\hline Chunjiang15 & TR & $4.87 b c$ & $4.90 \mathrm{~b}$ & $27.69 \mathrm{a}$ & $21.41 \mathrm{~d}$ & $19.63 \mathrm{c}$ & $21.88 \mathrm{~b}$ & $50.10 \mathrm{c}$ & $52.92 \mathrm{bc}$ \\
\hline & MR & $4.98 \mathrm{ab}$ & $5.09 \mathrm{a}$ & $26.51 \mathrm{ab}$ & $25.07 b c$ & $23.11 \mathrm{a}$ & $22.72 b$ & $54.49 \mathrm{~b}$ & $57.78 \mathrm{a}$ \\
\hline & $\mathrm{BR}$ & $4.85 b c$ & $4.75 \mathrm{c}$ & $24.68 \mathrm{c}$ & $21.03 \mathrm{~d}$ & $22.81 \mathrm{~b}$ & $23.23 \mathrm{a}$ & $57.74 \mathrm{a}$ & $58.07 \mathrm{a}$ \\
\hline & Mean 1 & 4.90 & 4.91 & 26.29 & 22.50 & 21.85 & 22.61 & 54.11 & 56.26 \\
\hline & Mean 2 & & & & & & & & \\
\hline & $\mathrm{CV}$ & & & & $1 \%$ & & $2 \%$ & & $0 \%$ \\
\hline Xiushui11 & TR & $4.23 b$ & $4.31 \mathrm{a}$ & $29.00 \mathrm{a}$ & $28.61 \mathrm{ab}$ & $17.28 \mathrm{c}$ & $18.15 b$ & $36.12 \mathrm{c}$ & $37.74 \mathrm{c}$ \\
\hline & MR & $4.49 \mathrm{a}$ & $4.25 b$ & $28.48 \mathrm{ab}$ & $24.26 c$ & $18.68 b$ & $18.09 \mathrm{~b}$ & $40.09 \mathrm{~b}$ & $39.13 b$ \\
\hline & BR & $4.51 \mathrm{a}$ & $4.38 \mathrm{a}$ & $27.49 b$ & $19.73 \mathrm{~d}$ & $20.21 \mathrm{a}$ & $19.98 \mathrm{a}$ & $43.62 \mathrm{a}$ & $43.45 \mathrm{a}$ \\
\hline & Mean 1 & 4.41 & 4.31 & 28.32 & 24.20 & 18.72 & 18.74 & 39.94 & 40.11 \\
\hline & Mean 2 & & & & & & & & \\
\hline & $\mathrm{CV}$ & & & & $4 \%$ & & $4 \%$ & & $7 \%$ \\
\hline
\end{tabular}

PR: primary rachis; SR: secondary rachis; TR: top rachis; MR: middle rachis; BR: bottom rachis. The crossing column between TR and PR means TPR (top primary rachis), and the corresponding expressions were for TSR (top secondary rachis, TR-SR), MPR (middle primary rachis, MR-PR), MSR (middle secondary rachis, MR-SR), BPR (bottom primary rachis, BR-PR), and BSR (bottom secondary rachis, BR-SR), respectively. Mean 1 is the average of three different positions for PR or SR; Mean 2 is the average of all six different positions; $\mathrm{CV}$ is the coefficient of variation. The values followed by the same letters within a column are not significantly different in term of the same cultivar $(P>0.05)$

somewhat lower PA concentrations relative to those on SR and BR. This result suggested that the grains with heavier weights were generally lower in PA concentrations in a comparison of different positions within a panicle, which was opposite to the effect of grain positions on mineral concentrations. However, the extent of positional variation in PA concentrations was considerably smaller compared with that in grain weight and most of minerals, in terms of their small CVs and narrow-range in PA among different grain positions for all six rice genotypes presented here (Tables 2 and 3). 
The molar ratio of $\mathrm{PA} / \mathrm{Zn}$ was commonly considered as an important parameter for the evaluation of $\mathrm{Zn}$ bioavailability in cereal and legumes (Raboy et al., 1984). As shown in Table 3, the average molar ratio of $\mathrm{PA} / \mathrm{Zn}$ ranged from 18.73 (Xiushui11) to 27.35 (Xiushui63) among the six genotypes, and the extent of positional variation in the molar ratio of $\mathrm{PA} / \mathrm{Zn}$ was much larger than that in PA concentrations among different grains within a panicle, as reflected by their CVs. In general, PR and TR tended to have lower molar ratio of $\mathrm{PA} / \mathrm{Zn}$ relative to $\mathrm{SR}$ and $\mathrm{BR}$, which was almost consistent with the tendency of positional differences in PA concentration. This result implied that grain position was closely related to $\mathrm{Zn}$ bioavailability in rice grains, with relatively high $\mathrm{Zn}$ bioavailability noted for PR and TR within a panicle.

The average molar ratio of $\mathrm{PA} / \mathrm{Fe}$ ranged from 38.52 to 65.04 among all six genotypes, wider variation compared with that of average molar ratio of $\mathrm{PA} / \mathrm{Zn}$ (Table 3). Moreover, the extent of the variation in the molar ratio of $\mathrm{PA} / \mathrm{Fe}$ among different grain positions was also somewhat greater than that in the molar ratio of $\mathrm{PA} / \mathrm{Zn}$, despite the similar trend for difference between PR and SR for both molar ratios of $\mathrm{PA} / \mathrm{Fe}$ and $\mathrm{PA} / \mathrm{Zn}$.

\subsection{Correlations among $\mathrm{K}, \mathrm{Mg}, \mathrm{Na}, \mathrm{Ca}, \mathrm{Zn}, \mathrm{Fe}$, $\mathrm{Mn}, \mathrm{Cu}, \mathrm{PA}$ contents and grain weight}

The Pearson correlation coefficients among eight minerals, PA and grain weight were calculated for six different grain positions in a panicle (Table 4). There were highly positive correlations $(P<0.01)$ between $\mathrm{Zn}$ and $\mathrm{Fe}$, and statistically positive correlations $(P<0.05$ or $P<0.01)$ among $\mathrm{Ca}, \mathrm{Zn}, \mathrm{Fe}$, and $\mathrm{Cu}$, regardless of genotypes. However, the statistical significance varied greatly with rice genotype for most of their correlations among other mineral concentrations, and also among minerals, PA, and grain weight. Generally, there were positive correlations among eight minerals, and between minerals and grain weight, but negative correlations between minerals and PA content.

Table 5 presents the correlations among the eight minerals, PA and grain weight for six genotypes. The correlation coefficients among the minerals were statistically significant only for K-Ca $(r=0.833)$, but no significant correlations $(P>0.05)$ were observed among PA and minerals, except for PA-Mn $(r=0.818)$.
Furthermore, grain weight was poorly correlated with minerals and PA. This result implied that grain PA concentration was relatively independent of grain weight and most metal minerals for different rice genotypes.

\section{Discussion}

The yield potential of a rice cultivar is closely related to grain number per panicle and grain weight (Cheng et al., 2007). However, it was practically difficult to make a balance of grain number and grain weight for a rice panicle owing to the limiting assimilation supply from leaves (Mohapatra and Sahu, 1991). In the past two decades, the enhancement of yield level for newly released japonica cultivars was mostly attributable to the elevated grain number per panicle (Padmajarao, 1995), and there was a narrow variation of grain weight between modern japonica cultivars and landraces (He et al., 2011). In fact, the japonica cultivars now being widely planted in China were mainly characterized by compact and erect or semi-erect panicles with more grains per panicle (Cheng et al., 2007). On the other hand, the compactpanicle cultivars were often found to show a great variation in floret development and grain filling among different rachides within a panicle (Liu et al., 2005b; Wang et al., 2006); also there were dramatic differences in protein composition, amylose content, grain weight, and chalkiness among different positional grains (Cheng et al., 2007). In our current study, grain position significantly affected the concentrations of minerals in both vertical and horizontal axes of the rice panicle. The grains located on top primary and middle primary rachides were substantially higher than those on bottom secondary rachides and middle secondary rachides for all measured minerals (K, Mg, Na, Ca, Mn, Zn, Fe, and $\mathrm{Cu}$ ). This result was generally in agreement with several previous findings, which demonstrated that the levels of $\mathrm{Zn}$ and $\mathrm{Fe}$ concentrations in wheat grain decreased at grain positions far distal from rachis (Calderini and OrtizMonasterio, 2003), and the heavy-weight grains had higher $\mathrm{Zn}$ and $\mathrm{Fe}$ concentrations relative to the small-weight grains within a wheat spike (Liu et al., 2006). However, little association was found between mineral concentrations and the rice yield for the rice 
Table 4 Correlation coefficients of $\mathrm{K}, \mathrm{Mg}, \mathrm{Na}, \mathrm{Ca}, \mathrm{Zn}, \mathrm{Fe}, \mathrm{Mn}, \mathrm{Cu}$, phytic acid (PA), and grain weight (GW) among different positions within a panicle

\begin{tabular}{|c|c|c|c|c|c|c|c|c|c|c|c|}
\hline \multirow{2}{*}{ Cultivar } & \multirow{2}{*}{ Feature } & \multicolumn{10}{|c|}{ Correlation coefficient } \\
\hline & & $\mathrm{K}$ & $\mathrm{Mg}$ & $\mathrm{Na}$ & $\mathrm{Ca}$ & $\mathrm{Zn}$ & $\mathrm{Fe}$ & $\mathrm{Mn}$ & $\mathrm{Cu}$ & PA & GW \\
\hline \multirow[t]{10}{*}{98110} & $\mathrm{~K}$ & 1.000 & & & & & & & & & \\
\hline & $\mathrm{Mg}$ & $0.960^{* *}$ & 1.000 & & & & & & & & \\
\hline & $\mathrm{Na}$ & $0.932^{* *}$ & $0.889^{*}$ & 1.000 & & & & & & & \\
\hline & $\mathrm{Ca}$ & $0.953^{* *}$ & $0.891^{*}$ & $0.991^{* *}$ & 1.000 & & & & & & \\
\hline & $\mathrm{Zn}$ & $0.904^{*}$ & 0.810 & $0.959^{* *}$ & $0.981^{* *}$ & 1.000 & & & & & \\
\hline & $\mathrm{Fe}$ & $0.856^{*}$ & 0.796 & $0.936^{* *}$ & $0.955^{* *}$ & $0.981^{* *}$ & 1.000 & & & & \\
\hline & $\mathrm{Mn}$ & -0.302 & -0.430 & -0.151 & -0.092 & 0.064 & 0.143 & 1.000 & & & \\
\hline & $\mathrm{Cu}$ & $0.874^{*}$ & 0.777 & $0.962^{* *}$ & $0.971^{* *}$ & $0.993^{* *}$ & $0.970^{* *}$ & 0.054 & 1.000 & & \\
\hline & PA & -0.577 & -0.353 & -0.662 & -0.677 & -0.730 & -0.612 & -0.117 & -0.768 & 1.000 & \\
\hline & GW & 0.702 & 0.663 & 0.711 & 0.770 & 0.794 & $0.854^{*}$ & 0.361 & 0.733 & -0.315 & 1.000 \\
\hline \multirow[t]{10}{*}{ HIPj } & $\mathrm{K}$ & 1.000 & & & & & & & & & \\
\hline & $\mathrm{Mg}$ & $0.963^{* *}$ & 1.000 & & & & & & & & \\
\hline & $\mathrm{Na}$ & 0.601 & 0.650 & 1.000 & & & & & & & \\
\hline & $\mathrm{Ca}$ & $0.816^{*}$ & $0.891^{*}$ & $0.880^{*}$ & 1.000 & & & & & & \\
\hline & $\mathrm{Zn}$ & $0.826^{*}$ & $0.886^{*}$ & $0.859^{*}$ & $0.990^{* *}$ & 1.000 & & & & & \\
\hline & $\mathrm{Fe}$ & 0.709 & 0.751 & $0.951^{* *}$ & $0.957^{* *}$ & $0.953^{* *}$ & 1.000 & & & & \\
\hline & $\mathrm{Mn}$ & $0.961^{* *}$ & $0.982^{* *}$ & 0.744 & $0.923^{* *}$ & $0.907^{*}$ & $0.825^{*}$ & 1.000 & & & \\
\hline & $\mathrm{Cu}$ & 0.743 & 0.753 & $0.883^{*}$ & $0.934^{* *}$ & $0.956^{* *}$ & $0.978^{* *}$ & $0.812^{*}$ & 1.000 & & \\
\hline & $\mathrm{PA}$ & 0.351 & 0.567 & 0.512 & 0.665 & 0.669 & 0.537 & 0.492 & 0.492 & 1.000 & \\
\hline & GW & 0.684 & 0.624 & $0.902^{*}$ & 0.744 & 0.729 & $0.839^{*}$ & 0.741 & 0.795 & 0.161 & 1.000 \\
\hline \multirow[t]{10}{*}{ Xiushui63 } & $\mathrm{K}$ & 1.000 & & & & & & & & & \\
\hline & $\mathrm{Mg}$ & $0.938^{* *}$ & 1.000 & & & & & & & & \\
\hline & $\mathrm{Na}$ & 0.746 & 0.541 & 1.000 & & & & & & & \\
\hline & $\mathrm{Ca}$ & 0.807 & 0.614 & $0.985^{* *}$ & 1.000 & & & & & & \\
\hline & $\mathrm{Zn}$ & $0.821^{*}$ & 0.651 & $0.953^{* *}$ & $0.972^{* *}$ & 1.000 & & & & & \\
\hline & $\mathrm{Fe}$ & 0.753 & 0.533 & $0.984^{* *}$ & $0.994^{* *}$ & $0.963^{* *}$ & 1.000 & & & & \\
\hline & $\mathrm{Mn}$ & $0.956^{* *}$ & $0.925^{* *}$ & 0.763 & 0.782 & 0.800 & 0.718 & 1.000 & & & \\
\hline & $\mathrm{Cu}$ & 0.780 & 0.618 & $0.947^{* *}$ & $0.963^{* *}$ & $0.881^{*}$ & $0.947^{* *}$ & 0.767 & 1.000 & & \\
\hline & $\mathrm{PA}$ & -0.473 & -0.531 & -0.483 & -0.533 & -0.661 & -0.503 & -0.477 & -0.433 & 1.000 & \\
\hline & GW & 0.789 & $0.836^{*}$ & 0.552 & 0.547 & 0.503 & 0.465 & $0.896^{*}$ & 0.634 & -0.201 & 1.000 \\
\hline \multirow[t]{10}{*}{ Xiushui994 } & $\mathrm{K}$ & 1.000 & & & & & & & & & \\
\hline & $\mathrm{Mg}$ & 0.669 & 1.000 & & & & & & & & \\
\hline & $\mathrm{Na}$ & $0.880^{*}$ & $0.853^{*}$ & 1.000 & & & & & & & \\
\hline & $\mathrm{Ca}$ & $0.848^{*}$ & $0.875^{*}$ & $0.978^{* *}$ & 1.000 & & & & & & \\
\hline & $\mathrm{Zn}$ & $0.879^{*}$ & 0.807 & $0.963^{* *}$ & $0.989^{* *}$ & 1.000 & & & & & \\
\hline & $\mathrm{Fe}$ & 0.772 & $0.819^{*}$ & $0.961^{* *}$ & $0.979^{* *}$ & $0.962^{* *}$ & 1.000 & & & & \\
\hline & $\mathrm{Mn}$ & 0.734 & $0.924^{* *}$ & $0.873^{*}$ & $0.852^{*}$ & 0.780 & $0.830^{*}$ & 1.000 & & & \\
\hline & $\mathrm{Cu}$ & $0.827^{*}$ & $0.865^{*}$ & $0.974^{* *}$ & $0.995^{* *}$ & $0.977^{* *}$ & $0.990^{* *}$ & $0.872^{*}$ & 1.000 & & \\
\hline & PA & 0.142 & -0.053 & 0.056 & -0.110 & -0.098 & -0.179 & -0.081 & -0.164 & 1.000 & \\
\hline & GW & 0.587 & $0.945^{* *}$ & $0.862^{*}$ & $0.909^{*}$ & $0.854^{*}$ & $0.898^{*}$ & $0.834^{*}$ & $0.905^{*}$ & -0.162 & 1.000 \\
\hline \multirow[t]{10}{*}{ Chunjiang15 } & $\mathrm{K}$ & 1.000 & & & & & & & & & \\
\hline & $\mathrm{Mg}$ & 0.690 & 1.000 & & & & & & & & \\
\hline & $\mathrm{Na}$ & 0.805 & 0.191 & 1.000 & & & & & & & \\
\hline & $\mathrm{Ca}$ & 0.802 & 0.460 & 0.607 & 1.000 & & & & & & \\
\hline & $\mathrm{Zn}$ & $0.869^{*}$ & 0.542 & 0.792 & $0.850^{*}$ & 1.000 & & & & & \\
\hline & $\mathrm{Fe}$ & $0.820^{*}$ & 0.488 & 0.641 & $0.993^{* *}$ & $0.881^{*}$ & 1.000 & & & & \\
\hline & $\mathrm{Mn}$ & $0.888^{*}$ & 0.618 & 0.655 & $0.967^{* *}$ & $0.933^{* *}$ & $0.971^{* *}$ & 1.000 & & & \\
\hline & $\mathrm{Cu}$ & $0.818^{*}$ & 0.480 & 0.715 & $0.944^{* *}$ & $0.949^{* *}$ & $0.972^{* *}$ & $0.956^{* *}$ & 1.000 & & \\
\hline & PA & 0.111 & -0.567 & 0.575 & 0.309 & 0.251 & 0.329 & 0.161 & 0.367 & 1.000 & \\
\hline & GW & 0.764 & 0.124 & $0.863^{*}$ & 0.592 & 0.615 & 0.567 & 0.602 & 0.540 & 0.439 & 1.000 \\
\hline \multirow[t]{10}{*}{ Xiushui11 } & $\mathrm{K}$ & 1.000 & & & & & & & & & \\
\hline & $\mathrm{Mg}$ & $0.871^{*}$ & 1.000 & & & & & & & & \\
\hline & $\mathrm{Na}$ & $0.921^{* *}$ & 0.690 & 1.000 & & & & & & & \\
\hline & $\mathrm{Ca}$ & $0.916^{*}$ & 0.751 & $0.938^{* *}$ & 1.000 & & & & & & \\
\hline & $\mathrm{Zn}$ & $0.944^{* *}$ & 0.733 & $0.887^{*}$ & $0.940^{* *}$ & 1.000 & & & & & \\
\hline & $\mathrm{Fe}$ & $0.909^{*}$ & 0.714 & $0.911^{*}$ & $0.990^{* *}$ & $0.970^{* *}$ & 1.000 & & & & \\
\hline & $\mathrm{Mn}$ & $0.842^{*}$ & 0.645 & $0.973^{* *}$ & $0.938^{* *}$ & 0.813 & $0.892^{*}$ & 1.000 & & & \\
\hline & $\mathrm{Cu}$ & $0.928^{* *}$ & 0.744 & $0.950^{* *}$ & $0.998^{* *}$ & $0.955^{* *}$ & $0.992^{* *}$ & $0.938^{* *}$ & 1.000 & & \\
\hline & PA & -0.337 & -0.189 & -0.507 & -0.503 & -0.458 & -0.500 & -0.548 & -0.522 & 1.000 & \\
\hline & GW & $0.838^{*}$ & $0.882^{*}$ & 0.709 & 0.804 & 0.734 & 0.771 & 0.700 & 0.782 & 0.041 & 1.000 \\
\hline
\end{tabular}

${ }^{* *},{ }^{*}$ Correlation is significant at the 0.01 and 0.05 levels (2-tailed), respectively 
Table 5 Correlation coefficients of $\mathrm{K}, \mathrm{Mg}, \mathrm{Na}, \mathrm{Ca}, \mathrm{Zn}, \mathrm{Fe}, \mathrm{Mn}, \mathrm{Cu}$, phytic acid (PA) and grain weight (GW) among six rice cultivars

\begin{tabular}{|c|c|c|c|c|c|c|c|c|c|c|}
\hline \multirow{2}{*}{ Feature } & \multicolumn{10}{|c|}{ Correlation coefficient } \\
\hline & $\mathrm{K}$ & $\mathrm{Mg}$ & $\mathrm{Na}$ & $\mathrm{Ca}$ & $\mathrm{Zn}$ & $\mathrm{Fe}$ & $\mathrm{Mn}$ & $\mathrm{Cu}$ & PA & GW \\
\hline $\mathrm{K}$ & 1.000 & & & & & & & & & \\
\hline $\mathrm{Mg}$ & 0.801 & 1.000 & & & & & & & & \\
\hline $\mathrm{Na}$ & 0.670 & 0.657 & 1.000 & & & & & & & \\
\hline $\mathrm{Ca}$ & $0.833^{*}$ & 0.403 & 0.270 & 1.000 & & & & & & \\
\hline $\mathrm{Zn}$ & -0.195 & 0.200 & -0.210 & -0.444 & 1.000 & & & & & \\
\hline $\mathrm{Fe}$ & -0.216 & -0.269 & 0.076 & -0.304 & 0.490 & 1.000 & & & & \\
\hline $\mathrm{Mn}$ & 0.724 & 0.621 & 0.699 & 0.597 & -0.611 & -0.562 & 1.000 & & & \\
\hline $\mathrm{Cu}$ & -0.573 & -0.759 & -0.590 & -0.112 & -0.318 & 0.036 & -0.236 & 1.000 & & \\
\hline PA & 0.562 & 0.726 & 0.568 & 0.285 & -0.339 & -0.744 & $0.818^{*}$ & -0.543 & 1.000 & \\
\hline GW & -0.420 & -0.133 & 0.316 & -0.661 & -0.209 & -0.057 & 0.153 & 0.030 & 0.285 & 1.000 \\
\hline
\end{tabular}

${ }^{* *},{ }^{*}$ Correlation is significant at the 0.01 and 0.05 levels (2-tailed), respectively

genotypes differing in grain density and grain weight (Table 2), and between grain weight and the mineral concentrations, in term of an average of six different grain positions (Table 5). The results indicated the feasibility of enhancing the desirable mineral concentrations in rice grains without causing reduction in grain weight and yield level by appropriate breeding strategies and agronomic management for the compact rice cultivars. Moreover, in light of the fact that the high yield potentials of compact rice cultivars were mostly achieved by high grain density and floret numbers in a panicle (Wang et al., 2006; Cheng et al., 2007), it might be practically efficient to increase grain mineral concentrations of whole rice panicles, with the variation of mineral concentration among grains within a panicle being simultaneously decreased, through the modification of their rachis patterns in a panicle, i.e., reducing grain number on the secondary rachis, or increasing grain number on the secondary rachis at the top rachis instead of at the bottom rachis.

Previous studies have showed that the enhancement of yield potentials could cause a significant reduction in nitrogen $(\mathrm{N})$ and phosphoros $(\mathrm{P})$ concentrations of wheat (Calderini et al., 1995), rice (Jongkaewwattana and Geng, 1991), maize (Simmonds, 1995), and sunflower grains (López Pereira et al., 2000). Additionally, initial findings indicated that grain weight was negatively related to grain PA, prolamin, glutelin, and total protein contents among different positions in a wheat spike (Herzog and Stamp, 1983; Ishimaru et al., 2005) or a rice panicle (Matsue et al., 1995; Liu et al., 2005b). Our present research concluded that the heavy-weight grains in a rice panicle generally had lower PA relative to the light ones (Table 3), which coincided well with previous reports (Liu et al., 2005b). Interestingly, our experimental results revealed that $\mathrm{Fe}$ and $\mathrm{Zn}$ concentrations of heavy-weight grains were generally higher than those of light-weight grains (Table 2), but the positional variations in $\mathrm{Fe}$ and $\mathrm{Zn}$ concentrations were poorly related to those in grain PA concentration for the different rachides within a rice panicle (Table 4). Thavarajah et al. (2010) investigated the impact of environmental temperature on $\mathrm{PA}, \mathrm{Zn}$ and $\mathrm{Fe}$ concentrations in lentil seeds, concluding that high PA content was independent of the increase in total $\mathrm{Zn}$ and $\mathrm{Fe}$ concentrations in lentil seeds for high temperature regimes. Furthermore, Iwai et al. (2012) revealed that total $\mathrm{Fe}, \mathrm{Zn}$, and $\mathrm{K}$ concentrations in filling grains increased constantly with rice grain development, and the well-developed mature grains had higher concentrations of $\mathrm{Fe}, \mathrm{Zn}$, and $\mathrm{K}$ minerals than the poorly-developed filling grains. According to our findings, the heavy-weight grains in a rice panicle generally had higher Fe and $\mathrm{Zn}$ concentrations relative to the small ones (Table 2). This trend was basically similar to the results reported previously by Thavarajah et al. (2010) and Iwai et al. (2012), but it was contradictory to a previous hypothesis, which 
presumed that the low $\mathrm{N}$ and $\mathrm{P}$ concentrations in heavy-weight grains were partially caused by the dilution of additional starch accumulation to other chemical components (Calderini and Ortiz-Monasterio, 2003). Nevertheless, these discordant phenomena did not exclude the possibility that grain $\mathrm{N}$ and $\mathrm{P}$ concentrations were quite different from many metal minerals in their positional variations within a panicle/ spike, which could be caused by their difference in the translocation and accumulation in the rice plant and grain location (Schachtman and Barker, 1999; Vasconcelos et al., 2003). Considering the extent of variation in grain mineral concentration among different positions within a rice panicle, the effect of grain position was more substantial for $\mathrm{Na}, \mathrm{Fe}$, and $\mathrm{Cu}$ than for $\mathrm{Ca}$ and $\mathrm{Zn}$ (Table 2). For rice breeding strategies, the different sensitivities to the effect of grain position on these metal minerals suggested that the improvement of panicle architecture and morphological pattern should be attended to more carefully if the deficiency of desired minerals was caused by low $\mathrm{Fe}$ concentration rather than low $\mathrm{Zn}$ and $\mathrm{Ca}$ concentrations in a compact rice cultivar. However, it should be stressed that the effect of grain position on the concentrations of PA and metal minerals (e.g., Fe, $\mathrm{Zn}$, and $\mathrm{Ca}$ ) was somewhat variable, depending on rice cultivars, and also the extent of positional variations in PA and metal minerals within a panicle was, in many cases, not so great as the cultivar-dependent difference in PA and metal minerals (Tables 2 and 3). For instance, Xiushuil1 had relatively higher $\mathrm{Fe}$, but inherently lower $\mathrm{Ca}$ concentration in rice grains than Chunjiang15, irrespective of grain position in the rice panicle. Thus, the selection of rice genotypes with low PA and high desirable minerals was an effective approach to directly reduce grain PA and to enhance the desirable mineral concentrations in rice grains for the improvement of its nutritional quality.

The inhibitory effect of PA on mineral absorption can be estimated by molar ratios of PA/mineral concentrations. The molar ratio of $\mathrm{PA} / \mathrm{Zn}$ was most widely applied to evaluate $\mathrm{Zn}$ bioavailability for human and animal absorption (Raboy et al., 1984). The molar ratios of $\mathrm{PA} / \mathrm{Zn}$ for cereals and legumes, maize, wheat, sorghum, and rice ranged approximately from 20 to 40 , and were relatively high compared with millet and soybean. In our study, the molar ratio of $\mathrm{PA} / \mathrm{Zn}$ in brown rice grains was very close to those reviewed by Adeyeye et al. (2000), but it was slightly lower than the previous report of Wei et al. (2012), possibly owing to differences in PA and mineral determination methods. Considering the variations of the molar ratios of $\mathrm{PA} / \mathrm{Zn}$ and $\mathrm{PA} / \mathrm{Fe}$ among different grain positions, $P R$ and TR generally were lower in both the molar ratios of $\mathrm{PA} / \mathrm{Zn}$ and $\mathrm{PA} / \mathrm{Fe}$ compared with BR and SR (Table 3), and also there was a significantly positive association between $\mathrm{Zn}$ and $\mathrm{Fe}$ concentrations for different grain positions within a panicle (Table 4) and positive correlation for various rice cultivars (Table 5). Our result was in agreement with the previous finding that transgenic rice with the ferritin gene enhanced $\mathrm{Zn}$ accumulation in rice grains (Vasconcelos et al., 2003). Therefore, it could be concluded that grain position had a large impact not only on grain $\mathrm{Zn}$ and $\mathrm{Fe}$ concentrations, but also on their bioavailability. Meanwhile, the lower $\mathrm{PA}$ and higher $\mathrm{Zn}$ or Fe concentrations for the heavier weight grains within a panicle implied that grain $\mathrm{Zn}$ and $\mathrm{Fe}$ concentrations in rice grains and their bioavailability could be concomitantly enhanced by a selection of good panicle architecture and morphological pattern for the rice genotypes, particularly for the compact rice genotypes with high grain density and yield levels.

\section{Compliance with ethics guidelines}

Da SU, Faisal SULTAN, Ning-chun ZHAO, Bing-ting LEI, Fu-biao WANG, Gang PAN, and Fang-min CHENG declare that they have no conflict of interest.

This article does not contain any studies with human or animal subjects performed by any of the authors.

\section{References}

Adeyeye, E.I., Arogundade, L.A., Akintayo, E.T., et al., 2000. Calcium, zinc and phytate interrelationship in some foods of major consumption in Nigeria. Food Chem., 71(4): 435-441. [doi:10.1016/S0308-8146(00)00159-X]

Anandan, A., Rajiv, G., Eswaran, R., et al., 2011. Genotypic variation and relationships between quality traits and trace elements in traditional and improved rice (Oryza sativa L.) genotypes. J. Food Sci., 76(4):H122-H130. [doi:10.1111/j.1750-3841.2011.02135.x]

Calderini, D.F., Ortiz-Monasterio, I., 2003. Grain position affects grain macronutrient and micronutrient concentrations in wheat. Crop Sci., 43(1):141-151. [doi:10.2135/ cropsci2003.0141]

Calderini, D.F., Torresleon, S., Slafer, G.A., 1995. Consequences of wheat breeding on nitrogen and phosphorus yield, grain nitrogen and phosphorus concentration and 
associated traits. Ann. Bot. (London), 76(3):315-322. [doi:10.1006/anbo.1995.1101]

Cheng, F.M., Liu, Y., Liu, Z.H., et al., 2007. Positional variation in chalky occurrence within a rice panicle and its relation to grain nutritional quality. Aust. J. Agric. Res., 58(2):95-103.

Ferguson, E.L., Gibson, R.S., Thompson, L.U., et al., 1988. Phytate, zinc, and calcium contents of 30 East African foods and their calculated phytate:Zn, Ca:phytate and [Ca][phytate]/[Zn] molar ratios. J. Food Compos. Anal., 1(4):316-325. [doi:10.1016/0889-1575(88)90031-2]

Frank, T., Habernegg, R., Yuan, F.J., et al., 2009. Assessment of the contents of phytic acid and divalent cations in low phytic acid (lpa) mutants of rice and soybean. J. Food Compos. Anal., 22(4):278-284. [doi:10.1016/j.jfca.2008. $11.022]$

He, X.H., Sun, Y., Gao, D., et al., 2011. Comparison of agronomic traits between rice landraces and modern varieties at different altitudes in the paddy fields of Yuanyang terrace, Yunnan province. J. Resour. Ecol., 2(1):46-50. [doi:10.3969/j.issn.1674-764x.2011.01.007]

Herzog, H., Stamp, P., 1983. Dry matter and nitrogen accumulation in grains at different ear positions in 'gigas', semidwarf and normal spring wheats. Euphytica, 32(2): 511-520. [doi:10.1007/BF00021463]

Ishimaru, T., Hirose, T., Matsuda, T., et al., 2005. Expression patterns of genes encoding carbohydrate-metabolizing enzymes and their relationship to grain filling in rice (Oryza sativa L.) comparison of caryopses located at different positions in a panicle. Plant Cell Physiol., 46(4):620-628. [doi:10.1093/pcp/pci066]

Iwai, T., Takahashi, M., Oda, K., et al., 2012. Dynamic changes in the distribution of minerals in relation to phytic acid accumulation during rice seed development. Plant Physiol., 160(4):2007-2014. [doi:10.1104/pp.112. 206573]

Jeng, T.L., Tseng, T.H., Wang, C.S., et al., 2006. Yield and grain uniformity in contrasting rice genotypes suitable for different growth environments. Field Crop Res., 99(1): 59-66. [doi:10.1016/j.fcr.2006.03.005]

Jongkaewwattana, S., Geng, S., 1991. Effect of nitrogen and water management on panicle development and milling quality of California rice (Oryza sativa L.). J. Agron. Crop Sci., 167(1):43-52. [doi:10.1111/j.1439-037X.1991. tb00932.x]

Kobayasi, K., Horie, Y., Imaki, T., 2002. Relationship between apical dome diameter at panicle initiation and the size of panicle components in rice grown under different nitrogen conditions during the vegetative stage. Plant Prod. Sci., 5(1):3-7. [doi:10.1626/pps.5.3]

Liu, Z.H., Cheng, F.M., Zhang, G.P., 2005a. Grain phytic acid content as affected by cultivar and environment and its relation to protein content. Food Chem., 89(1):49-52. [doi:10.1016/j.foodchem.2004.01.081]

Liu, Z.H., Cheng, F.M., Cheng, W.D., et al., 2005b. Positional variations in phytic acid and protein content within a panicle of japonica rice. J. Cereal Sci., 41(3):297-303. [doi:10.1016/j.jcs.2004.09.010]

Liu, Z.H., Wang, H.Y., Wang, X.E., et al., 2006. Genotypic and spike positional difference in grain phytase activity, phytate, inorganic phosphorus, iron, and zinc contents in wheat (Triticum aestivum L.). J. Cereal Sci., 44(2): 212-219. [doi:10.1016/j.jcs.2006.06.001]

López Pereira, M., Trápani, N., Sadras, V.O., 2000. Genetic improvement of sunflower in Argentina between 1930 and 1995: Part III. Dry matter partitioning and grain composition. Field Crop Res., 67(3):215-221. [doi:10. 1016/S0378-4290(00)00096-4]

Matsue, Y., Odahara, K., Hiramatsu, M., 1995. Differences in amylose content, amylographic characteristics and storage proteins of grains on primary and secondary rachis branches in rice (Oryza Sativa). Jpn. J. Crop Sci., 64(3): 601-606. [doi:10.1626/jcs.64.601]

McLaughlin, M.J., Parker, D.R., Clarke, J.M., 1999. Metals and micronutrients - food safety issues. Field Crop Res., 60(1-2):143-163. [doi:10.1016/S0378-4290(98)00137-3]

Miller, G.A., Youngs, V.L., Oplinger, E.S., 1980. Environmental and cultivar effects on oat phytic acid concentration. Cereal Chem., 57(3):189-191.

Mohapatra, P.K., Sahu, S.K., 1991. Heterogeneity of primary branch development and spikelet survival in rice panicle in relation to assimilates of primary branches. J. Exp. Bot., 42(7):871-879. [doi:10.1093/jxb/42.7.871]

Padmajarao, S., 1995. Yield and high density grain as influenced by crop density and $\mathrm{N}$ levels in scented rice. Madras Agric. J., 82(2):108-112.

Raboy, V., 2001. Seeds for a better future: 'low phytate' grains help to overcome malnutrition and reduce pollution. Trends Plant Sci., 6(10):458-462. [doi:10.1016/S13601385(01)02104-5]

Raboy, V., Dickinson, D.B., Below, F.E., 1984. Variation in seed total phosphorus, phytic acid, zinc, calcium, magnesium, and protein among lines of Glycine max and $G$. soja. Crop Sci., 24(3):431-434. [doi:10.2135/cropsci1984. 0011183X002400030001x]

Schachtman, D.P., Barker, S.J., 1999. Molecular approaches for increasing the micronutrient density in edible portions of food crops. Field Crops Res., 60(1-2):81-92. [doi:10. 1016/S0378-4290(98)00134-8]

Simmonds, N.W., 1995. The relation between yield and protein in cereal grain. J. Sci. Food Agric., 67(3):309-315. [doi:10.1002/jsfa.2740670306]

Thavarajah, D., Thavarajah, P., See, C.T., et al., 2010. Phytic acid and $\mathrm{Fe}$ and $\mathrm{Zn}$ concentration in lentil (Lens culinaris L.) seeds is influenced by temperature during seed filling period. Food Chem., 122(1):254-259. [doi:10.1016/j. foodchem.2010.02.073]

Umeta, M., West, C.E., Fufa, H., 2005. Content of zinc, iron, calcium and their absorption inhibitors in foods commonly consumed in Ethiopia. J. Food Compos. Anal., 18(8):803-817. [doi:10.1016/j.jfca.2004.09.008]

Vasconcelos, M., Datta, K., Oliva, N., et al., 2003. Enhanced 
iron and zinc accumulation in transgenic rice with the ferritin gene. Plant Sci., 164(3):371-378. [doi:10.1016/ S0168-9452(02)00421-1]

Wang, F., Cheng, F.M., Zhang, G.P., 2006. The relationship between grain filling and hormone content as affected by genotype and source-sink relation. Plant Growth Regul., 49(1):1-8. [doi:10.1007/s10725-006-0017-3]
Wei, Y.Y., Shohag, M.J., Wang, Y., et al., 2012. Effect of zinc sulfate fortification in germinated brown rice on seed zinc concentration, bioavailability, and seed germination. $J$. Agric. Food Chem., 60(7):1871-1879. [doi:10.1021/ jf205025b]

\section{中文概要:}

\section{本文题目：水稻穗内不同粒位间的矿质营养变化差异及其与籽粒植酸含量的关系}

Positional variation in grain mineral nutrients within a rice panicle and its relation to phytic acid concentration

研究目的：阐明水稻穗内不同粒位间的主要矿质营养元素和植酸含量差异、粒位分布特点及其与品种 穗型间的联系。

创新要点: 将水稻品种的穗型变化与稻米营养品质结合起来, 从水稻穗粒结构角度, 对同一稻穗内不 同籽粒间的主要矿质营养元素与植酸含量差异、粒位分布特点及其与水稻品种穗型间的相 互关系进行了较系统的探讨分析。

研究方法: 以典型的直立穗型和弯穗型粳稻品种为材料, 通过对两类水稻品种在相同栽培条件下籽粒 矿质营养元素和植酸含量的测定分析，并依据水稻籽粒在稻穗上的着生部位，将同一稻穗 内的不同籽粒划分为六个粒位，比较分析了两类品种同一稻穗内不同部位间矿质营养元素 和植酸含量的差异变化及其粒位分布特点。

重要结论: 水稻穗型虽然与品种间的籽粒矿质营养元素和植酸含量高低没有直接关系, 但对其穗内不 同籽粒间的主要矿质营养元素和植酸含量存在着较大影响; 与稻穗中下部的弱势粒相比, 同一稻穗内着生在稻穗上中部的强势粒通常具有相对较高的锌、铁矿质元素含量, 而籽粒 植酸含量和植酸/锌（铁）摩尔比则有所降低，稻米营养品质也相对较好；不同矿质营养元 素相比, 粒位效应对铁矿质营养的影响作用要略大于对钻和锌营养元素含量。

关键词组: 水稻穗型; 粒位效应; 营养品质; 矿质元素; 植酸 